\title{
A note on limit Results for the Penrose-BanzhaF INDEX
}

\author{
Sascha Kurz \\ Dept. of Mathematics, University of Bayreuth, Germany, sascha.kurz@uni-bayreuth.de
}

\begin{abstract}
It is well known that the Penrose-Banzhaf index of a weighted game can differ starkly from corresponding weights. Limit results are quite the opposite, i.e., under certain conditions the power distribution approaches the weight distribution. Here we provide parametric examples that give necessary conditions for the existence of limit results for the Penrose-Banzhaf index.
\end{abstract}

Keywords: weighted voting $\cdot$ power measurement $\cdot$ Penrose-Banzhaf index $\cdot$ limit results JEL codes: $\mathrm{C} 61 \cdot \mathrm{C} 71$

\section{Introduction}

Consider a private limited company with four shareholders. Assume that the shares are given by $(0.42,0.40,0.09,0.09)$ and that decisions are drawn by simple majority rule. The shares suggest that the influence on company decisions is similar for the first two and the last two shareowners. However, a proposal can be enforced either by shareholders 2,3, 4 or by shareholder 1 with the support of at least one of the others. Thus, restricting the analysis to shares and the decision rule, the three later shareowners have equal say, which is not reflected by the magnitude of shares at all. In order to evaluate influence in such decision environments, power indices like the Penrose-Banzhaf index [1, 9], the Shapley-Shubik index [12], or the nucleolus [1] were introduced. In our example the corresponding power distributions are given by $\left(\frac{1}{2}, \frac{1}{6}, \frac{1}{6}, \frac{1}{6}\right),\left(\frac{1}{2}, \frac{1}{6}, \frac{1}{6}, \frac{1}{6}\right)$, and $\left(\frac{2}{5}, \frac{1}{5}, \frac{1}{5}, \frac{1}{5}\right)$, respectively. So, our example is just an instance of the well known fact that relative weights can differ starkly from the corresponding power distribution. However, under certain conditions, weights and power are almost equal, which is studied under the term limit results for power indices in the literature. An early example was mentioned by Penrose in 1952, see the appendix of [9]. Roughly speaking, if a certain quantity, now known as the Laakso-Taagepera index [5] or Herfindahl-Hirschman index, is large, then for simple majority the weights are a good approximation for the Penrose-Banzhaf index. For a specific interpretation of the term "good approximation", a proof of some special cases and counter examples have been given in [6] and [7], respectively. Here we study a wider range of measures for deviation and provide parametric examples that give necessary conditions for the existence of limit results for the Penrose-Banzhaf index.

The remaining part of the paper is structured as follows. After introducing the necessary preliminaries in Section 2, we present our main results in Section 3 and set them into context. Auxiliary results and lengthy proofs are moved to an appendix.

\section{Preliminaries}

For a positive integer $n$ let $N=\{1, \ldots, n\}$ be the set of players. A simple game is a mapping $v: 2^{N} \rightarrow\{0,1\}$ with $v(\emptyset)=0, v(N)=1$, and $v(S) \leq v(T)$ for all $S \subseteq T \subseteq N$. We call 
$S \subseteq N \backslash\{i\}$ an $i$-swing if $v(S)=0, v(S \cup\{i\})=1$ and denote the number of $i$-swings in $v$ by $\eta_{i}(v)$. Setting $\eta(v)=\sum_{i \in N} \eta_{i}(v)$, the Penrose-Banzhaf index of player $i$ is given by $\operatorname{BZI}_{i}(v)=\eta_{i}(v) / \eta(v)$. The Shapley-Shubik index is given by the following weighted counting of swings

$$
\operatorname{SSI}_{i}(v)=\sum_{S \subseteq N \backslash\{i\}} \frac{|S| ! \cdot(n-|S|-1) !}{n !} \cdot(v(S \cup\{i\})-v(S)) .
$$

The nucleolus $\operatorname{Nuc}(v)$ can be defined as the unique solution of an optimization problem, see [11] for the details.

A simple game $v$ is weighted if there exists a quota $q \in \mathbb{R}_{>0}$ and weights $w \in \mathbb{R}_{\geq 0}^{n}$ such that $v(S)=1$ iff $w(S) \geq q$, where $w(S):=\sum_{i \in S} w_{i}$. We write $v=[q ; w]$ and speak of relative or normalized weights if $w(N)=1$. By $\Delta(w)=\max \left\{w_{i}: i \in N\right\}$ and $\Lambda(w)=\max \left\{w_{i} / w_{j}\right.$ : $\left.i, j \in N, w_{i}, w_{j} \neq 0\right\}$ we denote the maximum weight and the span (of weight vector $w$ ), respectively.

For a vector $x \in \mathbb{R}^{n}$ we write $\|x\|_{1}=\sum_{i=1}^{n}\left|x_{i}\right|,\|x\|_{\infty}=\max \left\{\left|x_{i}\right|: 1 \leq i \leq n\right\}$, and $\|x\|_{p}=\left(\sum_{i=1^{n}}\left|x_{i}\right|^{p}\right)^{1 / p}$ for $p \geq 1$. Each such norm $\|\cdot\|$ induces a distance function via $d(x, y)=\|x-y\|$. For all $x \in \mathbb{R}^{n}$ and all $1 \leq p \leq p^{\prime}$ we have $\|x\|_{\infty} \leq\|x\|_{p^{\prime}} \leq\|x\|_{p} \leq\|x\|_{1}$, i.e., $\|\cdot\|_{1}$ and $\|\cdot\|_{\infty}$ are the extreme cases on which we focus here. For normalized weight vectors, i.e., $w, w^{\prime} \in \mathbb{R}_{\geq 0}^{n}$ with $\|w\|_{1}=\left\|w^{\prime}\right\|_{1}=1$, the inequality $\left\|w-w^{\prime}\right\|_{\infty} \leq\left\|w-w^{\prime}\right\|_{1}$ can be strengthened to $\left\|w-w^{\prime}\right\|_{\infty} \leq\left\|w-w^{\prime}\right\|_{1} / 2$, see Lemma 4 .

\section{Approximation results}

Before we start to discuss approximation results between weights and the Penrose-Banzhaf index we briefly review the known results for the Shapley-Shubik index and the nucleolus. Neyman's main result of [8] implies as a special case:

Theorem 1. For each $\varepsilon>0$ there exist constants $\delta>0$ and $K>0$ such that for each $n \in \mathbb{N}$, $q \in(0,1)$ and $w \in \mathbb{R}^{n}$ with $\|w\|_{1}=1, \Delta(w)<\delta$, and $K \Delta(w)<q<1-K \Delta(w)$, we have $\|\mathrm{SSI}([q ; w])-w\|_{1}<\varepsilon$.

In words, the Shapley-Shubik index of a weighted game is close to relative weights if the maximum weight is small and the quota is not too near to the boundary points 0 or 1 . We remark that the maximum relative weight is small if and only if the Laakso-Taagepera index of $w$ is large, see Lemma 5 for the precise details. Invoking conditions on the maximum weight and the quota is indeed necessary for any power index $\varphi$.

Proposition 1. ([3, Proposition 1]) Let $\varphi$ be a mapping from the set of weighted games (on $n$ players) into $\mathbb{R}_{\geq 0}^{n}$.

(i) For each $q \in(0,1]$ and each integer $n \geq 2$ there exists a weighted game [q; $w]$, where $w \in \mathbb{R}_{\geq 0}^{n}$ and $\|w\|_{1}=1$, such that $\|w-\varphi([q ; w])\|_{1} \geq \frac{1}{3}$ and $\|w-\varphi([q ; w])\|_{\infty} \geq \frac{1}{6}$.

(ii) For each $\Delta \in(0,1)$ and each integer $n \geq \frac{4}{3 \Delta}+6$ there exists a weighted game [q; $\left.w\right]$, where $q \in(0,1], w \in \mathbb{R}_{\geq 0}^{n},\|w\|_{1}=1$, and $\Delta(w)=\Delta$, such that $\|w-\varphi([q ; w])\|_{1} \geq \frac{1}{3}$, and $\|w-\varphi([q ; w])\|_{\infty} \geq \Delta / 4$. 
The underlying reason is that different representations of the same weighted game have to be mapped onto to same power vector, i.e., the diameter of the polytope of representations of a weighted game plays the key role, as exploited in [3].

While the functional dependence for $\delta$ and $K$ on $\varepsilon$ is hidden in the existence arguments of the proofs of [8], a more explicit statement for the nucleolus was obtained in [4]:

Theorem 2. For $q \in(0,1), w \in \mathbb{R}_{\geq 0}^{n}$ with $\|w\|_{1}=1$ we have $\|\operatorname{Nuc}([q ; w])-w\|_{1} \leq \frac{2 \Delta(w)}{\min \{q, 1-q\}}$.

For the Penrose-Banzhaf index an analog of Theorem 1 is impossible.

Proposition 2. Let

$$
v_{n}=[n^{3}+n^{2} ; 2 n^{2}, \overbrace{1, \ldots, 1}^{2 n^{3}}] .
$$

Then $\|w\|_{1}=1, v_{n}=\left[\frac{1}{2} ; w\right]$, and for $n \geq 11$ we have

$$
\left\|\operatorname{BZI}\left(v_{n}\right)-w\right\|_{1} \geq 2-\frac{4}{n} \quad \text { and } \quad\left\|\operatorname{BZI}\left(v_{n}\right)-w\right\|_{\infty} \geq 1-\frac{2}{n} .
$$

For any given constants $\delta$ and $K$ we can choose $n$ large enough such that $\Delta\left(v_{n}\right)<\delta$ and $K \Delta\left(v_{n}\right)<q<1-K \Delta\left(v_{n}\right)$ since $\Delta\left(v_{n}\right)=\frac{1}{n}$ and $q=\frac{1}{2}$. Thus, $\left\|\operatorname{BZI}\left(v_{n}\right)-w\right\|_{1}>\varepsilon$ for $\varepsilon<1$ and $n \geq 11$ sufficiently large.

Note that for any $x, x^{\prime} \in \mathbb{R}_{\geq 0}^{n}$ with $\|x\|_{1}=\left\|x^{\prime}\right\|_{1}=1$ we have $\left\|x-x^{\prime}\right\|_{\infty} \leq 1$ and $\left\|x-x^{\prime}\right\|_{\infty} \leq 2$, i.e., for large $n$ the disparity between $\operatorname{BZI}\left(v_{n}\right)$ and the stated relative weights is as large as it could be for arbitrary vectors.

For the Penrose-Banzhaf index we have the following limit theorem, see [6].

Theorem 3. Let $\widetilde{W}$ be a finite set of non-negative integers, $W \subseteq \widetilde{W}$ be a finite set of positive integers with greatest common divisor $1, \rho \in \mathbb{R}_{>0}$, and $\left(w_{i}\right)_{i \in \mathbb{N}}$ be a sequence with $w_{i} \in \widetilde{W}$ for all $i \in \mathbb{N}$ such that $\left\{i \in \mathbb{N}: w_{i} \in \widetilde{W} \backslash W\right\}$ is finite and $\sum_{i \in\left\{1 \leq j \leq n: w_{j}=a\right\}} w_{i} \geq \rho \cdot \sum_{1 \leq i \leq n} w_{i}$ for all $a \in W$ and all sufficiently large $n$. Then,

$$
\lim _{n \rightarrow \infty} \frac{\operatorname{BZI}_{i}\left(\left[\frac{1}{2}, \bar{w}^{(n)}\right]\right)}{\operatorname{BZI}_{j}\left(\left[\frac{1}{2}, \bar{w}^{(n)}\right]\right)}=\frac{w_{i}}{w_{j}}
$$

for all integers $i, j$ with $w_{i}, w_{j} \in W$, where $\bar{w}_{h}^{(n)}=w_{h} / \sum_{l=1}^{n} w_{l}$ denotes the relative weight.

Since Equation (1) is a statement about the ratio between the Penrose-Banzhaf indices of two players whose weights are attained infinitely often, it is trivially satisfied in the example of Proposition 2, So, we give another parametric example.

Proposition 3. Let

$$
v_{n}=[3 n^{3}+n^{2} ; \overbrace{2 n^{2}, \ldots, 2 n^{2}}^{2 n+1}, \overbrace{1, \ldots, 1}^{2 n^{3}}] .
$$

Relative weights for a relative quota of $\frac{1}{2}$ are given by $w=\left(2 n^{2}, \ldots, 2 n^{2}, 1, \ldots, 1\right) /\left(6 n^{3}+2 n^{2}\right)$, i.e., $\|w\|_{1}=1$ and $v_{n}=\left[\frac{1}{2} ; w\right]$. For $n \geq 11$ we have $\mathrm{BZI}_{1}\left(v_{n}\right) / \mathrm{BZI}_{2 n+2}\left(v_{n}\right) \geq 2.6^{n} /(2 n+1)$ and $\left\|\operatorname{BZI}\left(v_{n}\right)-w\right\|_{1} \geq \frac{1}{5}$. 
Here we only have two types of players which we call large and small. The weight fraction of the small players tends to $\frac{1}{3}$ as $n$ increases and the maximum relative weight tends to zero. Nevertheless the ratio between the Penrose-Banzhaf powers of large and small players grows exponentially faster than the ratio between their weights. Of course this does not contradict Theorem 3 since $W$ is assumed to be finite. It was also noted in [2, Section 10] that the Penrose-Banzhaf index of $[q ; w]$ can behave strangely if the span $\Lambda(w)$ grows without bound. To that end we state:

Conjecture 1. There exists a constant $C>0$ such that for each $w \in \mathbb{R}_{>0}^{n}$ with $\|w\|_{1}=1$ we have

$$
\left\|\mathrm{BZI}\left(\left[\frac{1}{2} ; w\right]\right)-w\right\|_{1} \leq C \cdot \Delta(w) \cdot \Lambda(w) .
$$

At this place it is appropriate to discuss the relation between approximation errors in the $\|\cdot\|_{1}$ norm and relative deviations as in Theorem 3 .

Lemma 1. Let $x, w \in \mathbb{R}_{\geq 0}^{n}$ with $x_{j}=x_{h}$ for all $1 \leq j, h \leq n$ with $w_{j}=w_{h}$.

(a) If $\|x-w\|_{1} \leq \varepsilon$, then

$$
1-\frac{\varepsilon}{\alpha_{i}} \leq \frac{x_{i}}{w_{i}} \leq 1+\frac{\varepsilon}{\alpha_{i}}
$$

for all $1 \leq i \leq n$ with $w_{i}>0$, where $S_{i}=\left\{1 \leq j \leq n: w_{i}=w_{j}\right\}$ and $\alpha_{i}=w\left(S_{i}\right)$.

(b) If $w_{i}, w_{j}, x_{i}, x_{j} \neq 0, \varepsilon_{i}, \varepsilon_{j} \in[0,1)$ with $1-\varepsilon_{i} \leq \frac{x_{i}}{w_{i}} \leq 1+\varepsilon_{i}$ and $1-\varepsilon_{j} \leq \frac{x_{j}}{w_{j}} \leq 1+\varepsilon_{j}$, then

$$
\frac{1-\varepsilon_{i}}{1+\varepsilon_{j}} \leq \frac{w_{i}}{w_{j}} \cdot \frac{x_{j}}{x_{i}} \leq \frac{1+\varepsilon_{i}}{1-\varepsilon_{j}} \quad \text { and } \quad\left|\frac{x_{i}}{w_{i}}-\frac{x_{j}}{w_{j}}\right| \leq \varepsilon_{i}+\varepsilon_{j} .
$$

Proof. Only part (a) is non-trivial. If $x_{i} / w_{i}>1+\varepsilon / \alpha_{i}$ or $x_{i} / w_{i}<1-\varepsilon / \alpha_{i}$ then

$$
\|x-w\|_{1} \geq \sum_{j \in S_{i}}\left|x_{j}-w_{j}\right|=\left|S_{i}\right| \cdot\left|x_{i}-w_{i}\right|>\left|S_{i}\right| \cdot w_{i} \cdot \varepsilon / \alpha_{i}=\varepsilon
$$

a contradiction.

So, if $\|x-w\|_{1}$ is small, then $x_{i} / w_{i}$ is near to 1 and $x_{i} / x_{j}$ is near to $w_{i} / w_{j}$ provided that the numbers are non-zero and the involved players each belong to a family of players with equal weights and non-vanishing weight share. Assumptions on $x$, i.e., non-negativity and symmetry, are rather mild and satisfied by any published power index. If true, Conjecture 1 would imply Theorem 3. Indeed a small relative deviation is a tighter assumption than a small $\|\cdot\|_{1}$ distance.

Lemma 2. Let $S \subseteq N=\{1, \ldots, n\}, \hat{\varepsilon}, \tilde{\varepsilon}, \varepsilon \in \mathbb{R}_{>0}$, and $x, w \in \mathbb{R}^{n}$ with $w(N) \leq 1, w(N \backslash S) \leq$ $\hat{\varepsilon}, x(N \backslash S) \leq \tilde{\varepsilon}$, and $1-\varepsilon \leq x_{i} / w_{i} \leq 1+\varepsilon$ for all $i \in S$, then $\|x-w\|_{1} \leq \hat{\varepsilon}+\tilde{\varepsilon}+\varepsilon$.

Proof. Let $S^{+}=\left\{i \in S: x_{i} \geq w_{i}\right\}$ and $S^{-}=\left\{i \in S: x_{i}<w_{i}\right\}$, then

$$
\begin{aligned}
\|x-w\|_{1} & =\sum_{i \in N \backslash\{i\}}\left|x_{i}-w_{i}\right|+\left(x\left(S^{+}\right)-w\left(S^{+}\right)\right)+\left(w\left(S^{-}\right)-x\left(S^{-}\right)\right) \\
& \leq w(N \backslash S)+x(N \backslash S)+w(S) \cdot \varepsilon \leq \hat{\varepsilon}+\tilde{\varepsilon}+\varepsilon .
\end{aligned}
$$


In words, if we assume a small relative deviation for all players except a subset of players with a small mass in terms of $x$ and $w$, then the $\|\cdot\|_{1}$ distance is small.

So far we have always assumed a relative quota of $q=\frac{1}{2}$ for the Penrose-Banzhaf index. For $q \in(0,1] \backslash \frac{1}{2}$ we can consider the weighted game $v_{n, q}=[q \cdot 3 n ; 2, \ldots, 2,1, \ldots, 1]$ with $n$ players of weight 2 and another $n$ players of weight 1 . For each quota $q$ there exists a constant $\varepsilon>0$ such that $\left\|\operatorname{BZI}\left(v_{n, q}\right)-\bar{w}_{n, q}\right\|_{1} \geq \varepsilon$ for all sufficiently large $n$, where $\bar{w}_{n, q}$ denotes the corresponding relative weight vector, see Proposition 4 in the appendix for a more refined statement. Lemma 2 implies that the ratio between the Penrose-Banzhaf power of players of weight 2 and players of weight 1 does not converge to 2 . This example also implies that we cannot have an upper bound of the form

$$
\|\operatorname{BZI}([q ; w])-w\|_{1} \leq \frac{C \cdot \Delta(w)^{\alpha} \cdot \Lambda(w)^{\beta}}{\min \{q, 1-q\}^{\gamma}}
$$

for each $q \in(0,1), w \in \mathbb{R}_{>0}^{n}$ with $\|w\|_{1}=1$, where $C, \alpha, \beta, \gamma \in \mathbb{R}_{>0}$ are arbitrary constants. So, there is little room for limit results for the Penrose-Banzhaf index for quotas $q \neq \frac{1}{2}$.

With respect to the Shapley-Shubik index we state:

Conjecture 2. For each $q \in(0,1)$ and $w \in \mathbb{R}_{>0}^{n}$ with $\|w\|_{1}=1$ we have

$$
\|\mathrm{SSI}([q ; w])-w\|_{1} \leq \frac{5 \Delta(w)}{\min \{q, 1-q\}} .
$$

We remark that Conjecture 2 is valid for all of our three parametric examples.

\section{References}

[1] J. F. Banzhaf III. Weighted voting doesn't work: a mathematical analysis. Rutgers Law Review, 19:317, 1964.

[2] P. Dubey and L. S. Shapley. Mathematical properties of the Banzhaf power index. Mathematics of Operations Research, 4(2):99-131, 1979.

[3] S. Kurz. Bounds for the diameter of the weight polytope. Submitted, 2018.

[4] S. Kurz, S. Napel, and A. Nohn. The nucleolus of large majority games. Economics Letters, 123(2):139-143, 2014.

[5] M. Laakso and R. Taagapera. Effective number of parties: A measure with application to Western Europe. Comparative Political Studies, 12(1):3-27, 1979.

[6] I. Lindner and M. Machover. LS Penrose's limit theorem: proof of some special cases. Mathematical Social Sciences, 47(1):37-49, 2004.

[7] I. Lindner and G. Owen. Cases where the Penrose limit theorem does not hold. Mathematical Social Sciences, 53(3):232-238, 2007.

[8] A. Neyman. Renewal theory for sampling without replacement. The Annals of Probability, pages 464-481, 1982.

[9] L. S. Penrose. On the objective study of crowd behaviour. HK Lewis, 1952.

[10] V. V. Petrov. Sums of independent random variables. Springer-Verlag, Berlin, Heidelberg, New York, 1975.

[11] D. Schmeidler. The nucleolus of a characteristic function game. SIAM Journal on Applied Mathematics, 17(6):1163-1170, 1969.

[12] L. S. Shapley and M. Shubik. A method for evaluating the distribution of power in a committee system. American Political Science Review, 48(3):787-792, 1954. 


\section{Appendix}

In order to prove Proposition 2 and Proposition 3 we need a small numerical estimate and a tightening of the general bound $\|x\|_{\infty} \leq\|x\|_{1}$ in our setting.

Lemma 3. For $n \geq 11$ we have $2 n^{3} / 2.6^{n} \leq \frac{1}{n}$.

Proof. Let $f(n)=2 n^{4} / 2.6^{n}$. Since $f^{\prime}(n)=-2 n^{3}(n \ln (2.6)-4) / 2.6^{n}$ and $n \ln (2.6)-4>0$ for $n>4.19$, we have $f^{\prime}(n)<0$. Thus, $f(n) \leq f(11)<1$ for $n \geq 11$.

Lemma 4. For $w, w^{\prime} \in \mathbb{R}_{\geq 0}^{n}$ with $\|w\|_{1}=\left\|w^{\prime}\right\|_{1}=1$, we have $\left\|w-w^{\prime}\right\|_{\infty} \leq \frac{1}{2}\left\|w-w^{\prime}\right\|_{1}$.

Proof. With $S:=\left\{1 \leq i \leq n \mid w_{i} \leq w_{i}^{\prime}\right\}$ and $A:=\sum_{i \in S}\left(w_{i}^{\prime}-w_{i}\right), B:=\sum_{i \in N \backslash S}\left(w_{i}-w_{i}^{\prime}\right)$, where $N=\{1, \ldots, n\}$, we have $A-B=0$ since $\|w\|_{1}=\left\|w^{\prime}\right\|_{1}$ and $w, w^{\prime} \in \mathbb{R}_{\geq 0}^{n}$. Thus, $\left\|w-w^{\prime}\right\|_{1}=2 A$ and $\left\|w-w^{\prime}\right\|_{\infty} \leq \max \{A, B\}=A$.

Proof of Proposition 2. We easily check $\|w\|_{1}=1$ and $v_{n}=\left[\frac{1}{2} ; w\right]$. For $v=[q ; k, 1, \ldots, 1]$ with $m$ times weight 1 we have $\eta_{1}(v)=\sum_{i=1}^{k}\left(\begin{array}{c}m \\ q-i\end{array}\right)$ and $\eta_{2}(v)=\left(\begin{array}{c}m-1 \\ q-1\end{array}\right)+\left(\begin{array}{c}m-1 \\ q-k-1\end{array}\right)$ so that

$$
\eta_{1}\left(v_{n}\right)=\sum_{i=1}^{2 n^{2}}\left(\begin{array}{c}
2 n^{3} \\
n^{3}+n^{2}-i
\end{array}\right) \geq\left(\begin{array}{c}
2 n^{3} \\
n^{3}
\end{array}\right)
$$

and

$$
\eta_{2}\left(v_{n}\right)=\left(\begin{array}{c}
2 n^{3}-1 \\
n^{3}+n^{2}-1
\end{array}\right)+\left(\begin{array}{c}
2 n^{3}-1 \\
n^{3}-n^{2}-1
\end{array}\right)=\left(\begin{array}{c}
2 n^{3}-1 \\
n^{3}+n^{2}-1
\end{array}\right)+\left(\begin{array}{c}
2 n^{3}-1 \\
n^{3}+n^{2}
\end{array}\right)=\left(\begin{array}{c}
2 n^{3} \\
n^{3}+n^{2}
\end{array}\right)
$$

using $q=n^{3}+n^{2}, k=2 n^{2}$, and $m=2 n^{3}$.

Since $\left(1+\frac{1}{n}\right)^{n}$ is monotonically increasing we have $\left(1+\frac{1}{n}\right)^{n} \geq 2.6$ for $n \geq 11$, so that

$$
\frac{\eta_{1}\left(v_{n}\right)}{\eta_{2}\left(v_{n}\right)} \geq \frac{\left(n^{3}+n^{2}\right) !\left(n^{3}-n^{2}\right) !}{\left(n^{3}\right) !\left(n^{3}\right) !}=\frac{\prod_{i=1}^{n^{2}} n^{3}+i}{\prod_{i=1}^{n^{2}} n^{3}+i-n^{2}} \geq\left(1+\frac{n^{2}}{n^{3}}\right)^{n^{2}}=\left(\left(1+\frac{1}{n}\right)^{n}\right)^{n} \geq 2.6^{n} .
$$

From

$$
\mathrm{BZI}_{1}\left(v_{n}\right)=\frac{\eta_{1}\left(v_{n}\right)}{\eta_{1}\left(v_{n}\right)+m \cdot \eta_{2}\left(v_{n}\right)}=1-\frac{m \cdot \eta_{2}\left(v_{n}\right)}{\eta_{1}\left(v_{n}\right)+m \cdot \eta_{2}\left(v_{n}\right)} \geq 1-m \cdot \frac{\eta_{2}\left(v_{n}\right)}{\eta_{1}\left(v_{n}\right)} \geq 1-\frac{2 n^{3}}{2.6^{n}},
$$

$w_{1}=\frac{1}{n+1} \leq \frac{1}{n}$, and $2 n^{3} / 2.6^{n} \leq \frac{1}{n}$ for $n \geq 11$, see Lemma 3 , we deduce

$$
\left\|\operatorname{BZI}\left(v_{n}\right)-w\right\|_{\infty} \geq\left|\operatorname{BZI}_{1}\left(v_{n}\right)-w_{1}\right| \geq 1-\frac{2}{n} .
$$

From Lemma 4 we then conclude $\left\|\operatorname{BZI}\left(v_{n}\right)-w\right\|_{1} \geq 2-\frac{4}{n}$.

Proof of Proposition 3. We easily check $\|w\|_{1}=1$ and $v_{n}=\left[\frac{1}{2} ; w\right]$. For players $1 \leq i \leq 2 n+1$ examples of swing coalitions are given by $n$ other players of weight $2 n^{2}$ and $n^{3}$ players of weight 1 , so that

$$
\eta_{i}\left(v_{n}\right) \geq\left(\begin{array}{c}
2 n \\
n
\end{array}\right) \cdot\left(\begin{array}{c}
2 n^{3} \\
n^{3}
\end{array}\right)
$$


For players of weight 1 , i.e., $2 n+2 \leq i \leq 2 n+1+2 n^{3}$, we have

$$
\begin{aligned}
\eta_{i}\left(v_{n}\right) & =\sum_{j=0}^{2 n+1}\left(\begin{array}{c}
2 n+1 \\
j
\end{array}\right) \cdot\left(\begin{array}{c}
2 n^{3}-1 \\
3 n^{3}+n^{2}-j \cdot 2 n^{2}-1
\end{array}\right) \\
& \leq(n+1) \cdot\left(\begin{array}{c}
2 n+1 \\
n
\end{array}\right) \cdot\left(\begin{array}{c}
2 n^{3}-1 \\
n^{3}-n^{2}-1
\end{array}\right)+(n+1) \cdot\left(\begin{array}{c}
2 n+1 \\
n
\end{array}\right) \cdot\left(\begin{array}{c}
2 n^{3}-1 \\
n^{3}+n^{2}-1
\end{array}\right) \\
& =(n+1) \cdot\left(\begin{array}{c}
2 n+1 \\
n
\end{array}\right) \cdot\left(\begin{array}{c}
2 n^{3} \\
n^{3}+n^{2}
\end{array}\right)=(2 n+1) \cdot\left(\begin{array}{c}
2 n \\
n
\end{array}\right) \cdot\left(\begin{array}{c}
2 n^{3} \\
n^{3}+n^{2}
\end{array}\right)
\end{aligned}
$$

Similar as in the proof of Proposition 2 we conclude

$$
\frac{\mathrm{BZI}_{1}\left(v_{n}\right)}{\mathrm{BZI}_{2 n+2}\left(v_{n}\right)}=\frac{\eta_{1}\left(v_{n}\right)}{\eta_{2 n+2}\left(v_{n}\right)} \geq \frac{2.6^{n}}{2 n+1}
$$

noting that $\eta_{1}\left(v_{n}\right)=\eta_{i}\left(v_{n}\right)$ for all $1 \leq i \leq 2 n+2$ and $\eta_{2 n+2}\left(v_{n}\right)=\eta_{i}\left(v_{n}\right)$ for all $2 n+2 \leq$ $2 n+1+2 n^{3}$ due to symmetry. With this we compute

$$
\begin{aligned}
\mathrm{BZI}_{1}\left(v_{n}\right)-w_{1} & =\frac{\eta_{1}\left(v_{n}\right)}{(2 n+1) \cdot \eta_{1}\left(v_{n}\right)+2 n^{3} \cdot \eta_{2 n+2}\left(v_{n}\right)}-\frac{1}{3 n+1} \\
& =\frac{1}{2 n+1} \cdot\left(1-\frac{2 n^{3} \cdot \eta_{2 n+2}\left(v_{n}\right)}{(2 n+1) \cdot \eta_{1}\left(v_{n}\right)+2 n^{3} \cdot \eta_{2 n+2}\left(v_{n}\right)}\right)-\frac{1}{3 n+1} \\
& \geq \frac{1}{2 n+1} \cdot\left(\frac{3}{10}-\frac{2 n^{3}}{2 n+1} \cdot \frac{\eta_{2 n+2}\left(v_{n}\right)}{\eta_{1}\left(v_{n}\right)}\right) \\
& \geq \frac{1}{2 n+1} \cdot\left(\frac{1}{2}-\frac{2 n^{3}}{2.6^{n}}\right) \geq \frac{1}{2 n+1} \cdot\left(\frac{3}{10}-\frac{1}{n}\right) \geq \frac{1}{2 n+1} \cdot \frac{1}{5}
\end{aligned}
$$

for $n \geq 11$ (using Lemma 3). Thus

$$
\left\|\operatorname{BZI}\left(v_{n}\right)-w\right\|_{1} \geq \sum_{i=1}^{2 n+1}\left|\mathrm{BZI}_{i}\left(v_{n}\right)-w_{i}\right|=(2 n+1) \cdot\left|\mathrm{BZI}_{1}\left(v_{n}\right)-w_{1}\right| \geq \frac{1}{5}
$$

The details for our briefly sketched last example from Section 3 are given by:

Proposition 4. For $n \in \mathbb{N}$ and $q \in[0,1]$ let

$$
v_{n, q}=[q \cdot 3 n ; \overbrace{2, \ldots, 2}^{n}, \overbrace{1, \ldots, 1}^{n}]
$$

with $n$ times weight 2 and $n$ times weight 1 . Relative weights for a relative quota of $q$ are given by $w_{n, q}=(2, \ldots, 2,1, \ldots, 1) /(3 n)$, i.e., $\left\|w_{n, q}\right\|_{1}=1$ and $v_{n, q}=\left[q ; w_{n, q}\right]$. Then the function $f(q):=\lim _{n \rightarrow \infty}\left\|\operatorname{BZI}\left(v_{n, q}\right)-w_{n, q}\right\|_{1}$ satisfies $f(q)=f(1-q) \in\left[0, \frac{1}{3}\right]$ for all $q \in[0,1]$ and is strictly monotonically increasing in $\left[\frac{1}{2}, 1\right]$.

We refrain from giving a rigorous proof. Symmetry around $q=\frac{1}{2}$, i.e., $f(q)=f(1-q)$ follows by considering the dual game. For a relative quota $q$ near 0 or near 1 all players are equivalent so that $\operatorname{BZI}\left(v_{n, q}\right)=\frac{1}{2 n} \cdot(1, \ldots, 1)$, which gives $f(0)=f(1)=\frac{1}{3}$. From [주, Theorem 
3.6] we conclude $f\left(\frac{1}{2}\right)=0$. In order to check the existence of the limit and monotonicity numerically we state

$$
\begin{aligned}
\eta_{1}\left(v_{n, q}\right) & =\sum_{i=0}^{n}\left(\begin{array}{c}
n \\
i
\end{array}\right) \cdot\left(\begin{array}{c}
n-1 \\
\lceil q \cdot 3 n\rceil-2 i-1
\end{array}\right) \\
\eta_{n+1}\left(v_{n, q}\right) & =\sum_{i=0}^{n-1}\left(\begin{array}{c}
n-1 \\
i
\end{array}\right) \cdot\left(\left(\begin{array}{c}
n \\
\lceil q \cdot 3 n\rceil-2 i-2
\end{array}\right)+\left(\begin{array}{c}
n \\
\lceil q \cdot 3 n\rceil-2 i-1
\end{array}\right)\right) \\
& =\sum_{i=0}^{n-1}\left(\begin{array}{c}
n-1 \\
i
\end{array}\right) \cdot\left(\begin{array}{c}
n+1 \\
\lceil q \cdot 3 n\rceil-2 i-1
\end{array}\right)
\end{aligned}
$$

noting that convergence is rather slow and requires high precision computations. We remark that error bounds in general local limit theorems for lattice distributions like e.g. [10, Theorem 2 in Chapter VII] are (inevitably) too weak in order to determine $f(q)$ analytically. While the summands of $\eta_{1}\left(v_{n, q}\right)$ and $\eta_{n+1}\left(v_{n, q}\right)$ are unimodal and quickly sloping outside a small neighborhood around the almost coinciding peaks, the intuitive idea to bound the sums in terms of their maximal summands is not too easy to pursue. The maximal summand is not attained at $i \approx q \cdot n$, as one could expect. Even approximating $\log _{2}\left(\begin{array}{l}n \\ k\end{array}\right)$ by $n \cdot H(k / n)$, where $H(p)=-p \log _{2}(p)-(1-p) \log _{2}(1-p)$ is the binary entropy of $p$, gives that the maximum summand is attained for $i \approx n \cdot g(q)$, where

$$
g(q)=\frac{\tilde{g}(q)^{\frac{1}{3}}}{12}-\frac{-3 q^{2}+3 q+\frac{1}{2}}{\tilde{g}(q)^{\frac{1}{3}}}+q
$$

and

$$
\tilde{g}(q)=-216 q^{3}+324 q^{2}-108 q+6 \sqrt{972 q^{4}-1944 q^{3}+864 q^{2}+108 q+6} .
$$

Numerically we can check that this fancy function $g$ satisfies $q \leq g(q) \leq 1.07 \cdot g(q)$ for all $\frac{1}{2} \leq q \leq 1$ and is, of course, symmetric to $q=\frac{1}{2}$.

Given the numerical results for $f(q)$ we can state that $\frac{8}{3} \cdot\left|q-\frac{1}{2}\right|^{3}$ and $\frac{1}{3}-H(q) / 3 \log _{2}(2)$ correspond to curves that look similar to $f(q)$ and have a rather small absolute error.

For $w \in \mathbb{R}_{\geq 0}^{n}$ with $w \neq 0$ the Laakso-Taagepera index is given by

$$
L(w)=\left(\sum_{i=1}^{n} w_{i}\right)^{2} / \sum_{i=1}^{n} w_{i}^{2}
$$

In general we have $1 \leq L(w) \leq n$. If the weight vector $w$ is normalized, then the formula simplifies to $L(w)=1 / \sum_{i=1}^{n} w_{i}^{2}$. Under the name "effective number of parties" the index is widely used in political science to measure party fragmentation, see, e.g., [5]. We observe the following relations between the maximum relative weight $\Delta=\Delta(w)$ and the Laakso-Taagepera index $L(w)$ : 
Lemma 5. [3, Lemma 3] For $w \in \mathbb{R}_{\geq 0}^{n}$ with $\|w\|_{1}=1$, we have

$$
\frac{1}{\Delta} \leq \frac{1}{\Delta(1-\alpha(1-\alpha) \Delta)} \leq L(w) \leq \frac{1}{\Delta^{2}+\frac{(1-\Delta)^{2}}{n-1}} \leq \frac{1}{\Delta^{2}}
$$

for $n \geq 2$, where $\alpha:=\frac{1}{\Delta}-\left\lfloor\frac{1}{\Delta}\right\rfloor \in[0,1)$. If $n=1$, then $\Delta=L(w)=1$.

Proof. The key idea is to optimize $\sum_{i=1}^{n} w_{i}^{2}$ with respect to the constraints $w \in \mathbb{R}^{n},\|w\|_{1}=1$, and $\Delta(w)=\Delta$.

For $n=1$, we have $w_{1}=1, \Delta(w)=1, \alpha=0$, and $L(w)=1$, so that we assume $n \geq 2$ in the remaining part of the proof. For $w_{i} \geq w_{j}$ consider $a:=\frac{w_{i}+w_{j}}{2}$ and $x:=w_{i}-a$, so that $w_{i}=a+x$ and $w_{j}=a-x$. With this we have $w_{i}^{2}+w_{j}^{2}=2 a^{2}+2 x^{2}$ and $\left(w_{i}+y\right)^{2}+\left(w_{j}-y\right)^{2}=2 a^{2}+2(x+y)^{2}$. Let us assume that $w^{\star}$ minimizes $\sum_{i=1}^{n} w_{i}^{2}$ under the conditions $w \in \mathbb{R}_{\geq 0},\|w\|_{1}=1$, and $\Delta(w)=\Delta$. (Since the target function is continuous and the feasible set is compact and non-empty, a global minimum indeed exists.) W.l.o.g. we assume $w_{1}^{\star}=\Delta$. If there are indices $2 \leq i, j \leq n$ with $w_{i}^{\star}>w_{j}^{\star}$, i.e., $x>0$ in the above parameterization, then we may choose $y=-x$. Setting $w_{i}^{\prime}:=w_{i}^{\star}+y=a=\frac{w_{i}^{\star}+w_{j}^{\star}}{2}, w_{j}^{\prime}:=w_{j}^{\star}-y=a=\frac{w_{i}^{\star}+w_{j}^{\star}}{2}$, and $w_{h}^{\prime}:=w_{h}^{\star}$ for all $1 \leq h \leq n$ with $h \notin\{i, j\}$, we have $w^{\prime} \in \mathbb{R}_{\geq 0}^{n},\left\|w^{\prime}\right\|_{1}=1, \Delta\left(w^{\prime}\right)=\Delta$, and $\sum_{h=1}^{n}\left(w_{h}^{\prime}\right)^{2}=\sum_{h=1}^{n}\left(w_{h}^{\star}\right)^{2}-x^{2}$. Since this contradicts the minimality of $w^{\star}$, we have $w_{i}^{\star}=w_{j}^{\star}$ for all $2 \leq i, j \leq n$, so that we conclude $w_{i}^{\star}=\frac{1-\Delta}{n-1}$ for all $2 \leq i \leq n$ from $1=\left\|w^{\star}\right\|_{1}=\sum_{h=1}^{n} w_{h}^{\star}$. Thus, $L(w) \leq 1 /\left(\Delta^{2}+\frac{(1-\Delta)^{2}}{n-1}\right)$, which is tight. Since $\Delta \leq 1$ and $n \geq 2$, we have $1 /\left(\Delta^{2}+\frac{(1-\Delta)^{2}}{n-1}\right) \leq \frac{1}{\Delta^{2}}$, which is tight if and only if $\Delta=1$, i.e., $n-1$ of the weights have to be equal to zero.

Now, let us assume that $w$ maximizes $\sum_{i=1}^{n} w_{i}^{2}$ under the conditions $w \in \mathbb{R}_{\geq 0},\|w\|_{1}=1$, and $\Delta(w)=\Delta$. (Due to the same reason a global maximum indeed exists.) Due to $1=$ $\|w\|_{1} \leq n \Delta$ we have $0<\Delta \leq 1 / n$, where $\Delta=1 / n$ implies $w_{i}=\Delta$ for all $1 \leq i \leq n$. In that case we have $L(w)=n$ and $\alpha=0$, so that the stated lower bounds for $L(w)$ are valid. In the remaining cases we assume $\Delta>1 / n$. If there would exist two indices $1 \leq i, j \leq n$ with $w_{i} \geq w_{j}, w_{i}<\Delta$, and $w_{j}>0$, we may strictly increase the target function by moving weight from $w_{j}$ to $w_{i}$ (this corresponds to choosing $y>0$ ), by an amount small enough to still satisfy the constraints $w_{i} \leq \Delta$ and $w_{j} \geq 0$. Since $\Delta>0$, we can set $a:=\lfloor 1 / \Delta\rfloor \geq 0$ with $a \leq n-1$ due to $\Delta>1 / n$. Thus, for a maximum solution, we have exactly $a$ weights that are equal to $\Delta$, one weight that is equal to $1-a \Delta \geq 0$ (which may indeed be equal to zero), and $n-a-1$ weights that are equal to zero. With this and $a \Delta=1-\alpha \Delta$ we have $\sum_{i=1}^{n} w_{i}^{2}=a \Delta^{2}(1-a \Delta)^{2}=\Delta-\alpha \Delta^{2}+\alpha^{2} \Delta^{2}=\Delta\left(1-\alpha \Delta+\alpha^{2} \Delta\right)=\Delta(1-\alpha(1-\alpha) \Delta) \leq \Delta$. Here, the latter inequality is tight if and only if $\alpha=0$, i.e., $1 / \Delta \in \mathbb{N}$. 